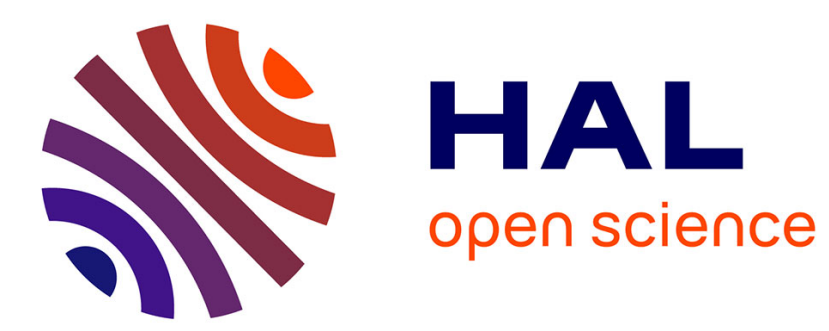

\title{
En résumé - Numéro 3-2013
}

- [.]revue Forestière Française, Rédaction

\section{- To cite this version:}

- [.]revue Forestière Française, Rédaction. En résumé - Numéro 3-2013. 2013, pp.287-292. 10.4267/2042/51860 . hal-03536087

\section{HAL Id: hal-03536087 \\ https://hal.science/hal-03536087}

Submitted on 19 Jan 2022

HAL is a multi-disciplinary open access archive for the deposit and dissemination of scientific research documents, whether they are published or not. The documents may come from teaching and research institutions in France or abroad, or from public or private research centers.
L'archive ouverte pluridisciplinaire HAL, est destinée au dépôt et à la diffusion de documents scientifiques de niveau recherche, publiés ou non, émanant des établissements d'enseignement et de recherche français ou étrangers, des laboratoires publics ou privés. 


\section{EN RESUME}

\section{Note de la rédaction}

Des thèses, des mémoires de fin d'études sont soutenus chaque année dans le domaine de la forêt et du bois. Il nous est apparu intéressant de faire connaître certains d'entre eux à nos lecteurs, dans cette rubrique intitulée "En résumé".

\section{Spatialisation du bilan hydrique des sols pour caractériser la distribution et la croissance des espèces forestières dans un contexte de changement climatique}

par Christian PIEDALLU

Thèse soutenue publiquement le 9 janvier 2012 à Nancy

Directeur de thèse : M. Jean-Claude Gégout (AgroParisTech, LERFoB)

De nombreuses recherches se focalisent sur l'étude des aires de distribution des espèces qui se décalent vers des conditions plus adaptées à leurs besoins physiologiques sous l'effet du changement climatique. Le choix des indices utilisés pour caractériser l'écologie des espèces et définir leur vulnérabilité au réchauffement en cours est souvent conditionné par leur disponibilité, alors qu'il devrait être basé sur les connaissances en écophysiologie qui les concernent. D'autre part, la résolution spatiale parfois grossière utilisée n'est pas toujours pertinente au regard de l'échelle à laquelle les processus biologiques se déroulent. Dans ce cadre, l'objectif de ce travail est de cartographier à fine résolution spatiale les bilans en eau des sols et leurs différentes composantes à l'échelle des forêts de France, et d'évaluer leur intérêt pour modéliser la distribution ou la productivité des espèces au regard des indices traditionnellement utilisés.

Dans un premier temps, nous avons modélisé et cartographié les différentes composantes du bilan en eau des sols, et tout particulièrement le rayonnement solaire et la réserve utile maximale en eau (RUM) des sols forestiers à partir des relevés de l'Inventaire forestier national (IFN). Ces données ont été combinées avec des températures et des précipitations pour spatialiser le bilan en eau des sols forestiers de France. Les principaux résultats montrent l'importance de la nébulosité dans la prise en compte du calcul du rayonnement solaire, et l'inefficacité des indices dérivés de l'exposition pour en simuler les valeurs à l'échelle de la France. Nous avons également déterminé qu'il est possible de réaliser avec des informations simples à collecter une carte des RUM des sols forestiers de France. Elle permet de prédire la croissance des essences avec une efficacité comparable aux valeurs relevées sur des placettes et d'améliorer la modélisation de la distribution de certaines essences. Enfin, nous démontrons que les calculs de bilans en eau qui prennent en compte la réserve en eau des sols sont plus efficaces que les bilans hydriques climatiques ou les pluies, particulièrement pour ce qui concerne les espèces hygrophiles ou xérophiles. Ces résultats laissent penser que l'importance de l'eau a été sous-estimée dans l'analyse de la distribution des espèces et l'étude des conséquences du changement climatique sur les plantes. Les données produites permettent de progresser dans la connaissance de l'écologie des espèces et de mieux caractériser la vulnérabilité des espèces, ouvrant la porte à la création d'outils plus fonctionnels pour aider les gestionnaires à évaluer les impacts du changement de climat et à s'y adapter. 


\section{Thyllose et perméabilité du bois des Chênes rouvre et pédonculé destiné à la fabrication des merrains, relation avec l'étanchéité des tonneaux}

par Naoual MELLOUKI-BENDIMRED

Thèse soutenue publiquement le 24 janvier 2012 à Nancy

Directeur de thèse : $M$. Patrick Perré (École centrale Paris, Laboratoire du génie des procédés et des matériaux) ; Co-directrice : Mme Marie-Christine Trouy-Triboulot (ENSTIB)

L'étanchéité des bois des Chênes sessile et pédonculé dans la direction longitudinale dépend de la quantité et de la qualité de la thyllose (la thyllose est la production de substances obstruant les vaisseaux âgés du bois). Depuis quelques années, un taux de fuite anormalement élevé dans les barriques fabriquées a été noté. Une étude expérimentale a été menée sur deux tonneaux non étanches et les fuites observées ont donné lieu à une exploration fine des voies de passage du liquide coloré à l'intérieur des douelles défectueuses. Nous avons pu mettre en relation une thyllose faible ou absente avec une migration de liquide qui peut s'effectuer sur toute la longueur d'une douelle. Par ailleurs, une production spéciale de merrains a été réalisée afin de comprendre l'influence de la hauteur dans l'arbre, de l'âge cambial, de l'arrosage et des différents paramètres de maturation des merrains (temps, position dans la palette) sur la perméabilité et la thyllose. Il a été nécessaire de mettre au point une méthode pour évaluer quantitativement la thyllose. Deux méthodes sont utilisées : la première repose sur l'observation anatomique et la propriété des vaisseaux non obstrués ou partiellement obstrués par des thylles de laisser filtrer la lumière. La seconde consiste à réaliser des mesures de la perméabilité à l'air sur des éprouvettes cylindriques taillées dans la direction longitudinale. L'infradensité des échantillons a été également mesurée. Les observations en microscopie électronique à balayage ont permis d'étudier la structure fine des thylles et de déceler la présence de spores et d'hyphes mycéliens. Les résultats sont analysés avec un logiciel de traitement statistique des données. Nous avons pu déterminer l'effet néfaste des développements microbiologiques, en particulier des champignons, sur la perméabilité longitudinale et la quantité de la thyllose du bois en cas de maturation trop longue.

\section{Variations spatio-temporelles de la réponse au climat des essences forestières tempérées : quantification du phénomène par approche dendroécologique et influence de la stratégie d'échantillonnage}

par Pierre MÉRIAN

Thèse soutenue publiquement le 2 mars 2012 à Nancy

Directeur de thèse : $M$. Jean-Claude Gégout (AgroParisTech, LERFoB) ; encadrant de thèse : M. François Lebourgeois (AgroParisTech, LERFoB)

En contexte tempéré, les études sur l'instabilité spatiotemporelle de la sensibilité des essences forestières au climat sont rares et souvent conduites à des échelles locales et régionales. De telles approches ne permettent pas d'obtenir une vision globale de la réponse à l'environnement et à ses variations. La fusion de jeux de données dendrochronologiques (plus de 4500 arbres carottés) a permis d'analyser le comportement de croissance de sept essences européennes tempérées majeures (Quercus petraea, Fagus sylvatica, Abies alba, Picea abies, Pinus sylvestris, Pinus nigra, Pinus uncinata) dans des contextes climatiques variés (océanique à subalpin) et sur l'ensemble $\mathrm{du} \mathrm{XX}^{\mathrm{e}}$ siècle. Les relations cerne-climat ont été évaluées par le calcul de fonctions de corrélation.

Les différences de protocoles de récolte des données des jeux agrégés ont également conduit à analyser la sensibilité des résultats aux variations de la stratégie d'échantillonnage. Nous proposons ici de quantifier l'effet de la taille (nombre d'arbres carottés) et des caractéristiques de l'échantillon (nombre de placettes, nombre d'arbres par placette, statuts sociaux couverts) sur la 
qualité de l'estimation du signal environnemental contenu dans la chronologie moyenne d'une part et d'autre part des fonctions de corrélation. Cette analyse a également permis de préciser dans quelle mesure cet effet " échantillon » était modulé par les différences de traits fonctionnels entre espèces et de contextes climatiques (plus ou moins limitants).

Quels que soient l'essence et le contexte écologique, la sécheresse estivale est le principal facteur limitant la croissance radiale (mais non le seul), suivie par la sécheresse de l'automne précédent et enfin le froid hivernal. La variabilité spatiale de la réponse dépend plus fortement de la pluviométrie que des températures, une pluviométrie faible conduisant à une sensibilité plus forte au froid hivernal et aux sécheresses estivale et automnale. Ce comportement général est modulé par les conditions écologiques locales, avec une sensibilité à la sécheresse moindre sur sol profond. Les différences interspécifiques s'expriment principalement hors saison de végétation (novembre à mars), même si les corrélations sont rarement significatives. La croissance des résineux est généralement stimulée par des fins d'hiver chaudes (février à avril), alors que celle des feuillus est corrélée négativement aux températures et positivement aux précipitations en décembre et janvier. Ces différences entre essences s'avèrent plutôt stables le long des gradients climatiques. Enfin, l'analyse temporelle révèle de fortes instabilités des relations cerneclimat au cours du siècle dernier. Le sens et l'ampleur de ces variations sont homogènes le long des gradients écologiques, mais en revanche peu synchrones avec les instabilités climatiques (automne, hiver, printemps) ou écophysiologiquement peu claires (été). Cette faible cohérence entre tendances climatiques et instabilité de la sensibilité au climat pourrait s'expliquer par l'absence d'une contrainte climatique de croissance unique en contexte tempéré, où la largeur de cerne est sous le double contrôle du froid hivernal et du stress hydrique estival (et automnal). Elle pourrait également provenir de phénomènes non climatiques, tels que l'effet biologique lié au vieillissement ou l'évolution progressive des pratiques de gestion forestière.

La taille et les caractéristiques de l'échantillon affectent le signal contenu dans la chronologie maîtresse de croissance et l'estimation des relations cerne-climat. Une diminution du nombre d'arbres carottés entraîne une estimation plus bruitée du signal environnemental commun à tous les arbres conduisant à une augmentation de l'erreur d'estimation des coefficients de corrélation bootstrap (BCC). Cette erreur se compose d'un biais équivalent à la racine carrée de l'expressed population signal qui conduit à une sous-estimation de la force des corrélations, et d'une dispersion élevée des estimations des BCC. Une conséquence de cette erreur croissante est l'augmentation du risque d'estimer des corrélations non significatives alors que celles-ci le sont à l'échelle de la population. La diminution du nombre de placettes affecte plus fortement la qualité des analyses dendroécologiques que la diminution du nombre d'arbres par placette. Enfin, de fortes différences ont été trouvées entre espèces et contextes écologiques. L'estimation des relations cerne-climat des essences tolérantes à l'ombrage (dites sciaphiles comme Fagus sylvatica, Abies alba, Picea abies) est plus sensible aux caractéristiques de l'échantillon que celle des essences héliophiles (Quercus petraea, Pinus sylvestris, Pinus nigra). Ainsi, pour une erreur donnée, le nombre d'arbres carottés doit être 40 à $60 \%$ plus élevé pour les essences sciaphiles que pour les essences héliophiles. Cette différence est attribuable à une plus forte variabilité inter-arbre des comportements de croissance pour les essences sciaphiles, notamment entre statuts sociaux, avec une sensibilité au climat d'autant plus forte que l'arbre est dominant. De même, la taille d'échantillon nécessaire pour atteindre une erreur acceptable diminue en contexte limitant (c'està-dire sous climat chaud et $\mathrm{sec}$, à haute altitude et sur sol peu profond). Nous proposons enfin une amélioration de la méthode d'évaluation des relations cerne-climat, basée sur le calcul de fonctions de corrélation multiples à partir de sous-échantillons issus d'une procédure de tirage aléatoire des arbres avec remise. Cette méthode permet de corriger le biais relatif à la taille de l'échantillon, d'estimer la dispersion des estimations et de réduire le risque de conclure à des corrélations non significatives alors que celles-ci le sont à l'échelle de la population. 


\section{Étude du comportement mécanique de matériaux isolants à base de fibres de bois : caractérisation à partir de techniques d'imagerie non destructives}

par Huyen TRAN

Thèse soutenue publiquement le 3 décembre 2012 à Bordeaux

Directeurs de thèse : Mme Christine Delisée (Bordeaux Sciences Agro), M. Pascal Doumalin (Institut Pprime Poitiers)

La thèse a pour but de caractériser les propriétés mécaniques de matériaux à base de fibres de bois en relation avec leur microstructure complexe. Ce type de matériau est hétérogène à différentes échelles : à l'échelle microscopique de la fibre, à l'échelle mésoscopique du réseau des fibres et à l'échelle macroscopique du matériau. Pour observer ces hétérogénéités, différents moyens expérimentaux de caractérisation sont utilisés, notamment la microtomographie aux rayons $X$ et la corrélation d'images volumiques. Ces deux techniques permettent à la fois de numériser les différentes fibres à l'échelle microscopique dans le volume, et d'obtenir le champ tridimensionnel de déformation à cœur. Comme résultats, le matériau étudié montre un comportement non linéaire avec une déformation résiduelle et un effet d'hystérésis en charge-décharge, qui suit le modèle de Van-Wyk. À l'échelle microscopique, le champ de déformation 3D fortement hétérogène est intimement lié aux porosités locales.

\section{Tomographie à rayons $\mathrm{X}$; analyse et modélisation de l'ontogénèse des épicormiques du Chêne sessile (Quercus petraea (L.) Matt.)}

par Jean-Baptiste MORISSET

Thèse soutenue publiquement le 11 décembre 2012 à Nancy

Directeur de thèse : $M$. Francis Colin (INRA, LERFoB)

Le Chêne sessile (Quercus petraea) est une essence majeure de la sylviculture française. Sa ramification, phénomène de mise en place des rameaux, a pour origine unique les bourgeons proventifs axillaires mis en place lors de l'élongation des pousses annuelles. On distingue la ramification séquentielle (les branches séquentielles) et la ramification épicormique (d'un mot grec signifiant : sur le tronc). La genèse des épicormiques, c'est-à-dire le passage d'un bourgeon épicormique à un rameau épicormique plus complexe tel qu'un gourmand, picot ou broussin est encore peu connue. Les rameaux laissent dans le bois des marques, les traces raméales, qui peuvent fortement déprécier la qualité du bois.

Étudier la formation des rameaux épicormiques du chêne passe par un suivi longitudinal ou une analyse rétrospective des pousses ou par une dissection de l'arbre et une observation des traces raméales. Pour nous affranchir de ces méthodologies, nous avons utilisé la tomographie à rayons $X$ qui permet une étude exhaustive de toutes les traces raméales d'un billon, de leur apparition ainsi que la reconstruction du billon en 3 dimensions. Les études menées au cours de cette thèse ont permis de mettre en évidence un important effet de l'arbre : chacun a sa propre capacité à mettre en place et maintenir des épicormiques. Plus un arbre présente d'épicormiques dans son jeune âge, plus il en présentera quelques années plus tard. Cette tendance est modulée par la sylviculture actuelle, comme le maintien d'une végétation d'accompagnement qui joue sur les réserves en eau et glucides de l'arbre, et la sylviculture passée, notamment les éclaircies fortes ayant favorisé les émissions de gourmands. Cependant la sélection précoce des arbres objectifs présentant le moins de formations épicormiques est une recommandation sylvicole de première importance dans le but de produire davantage de bois d'œuvre de la meilleure qualité possible. Une proposition d'intégration de ces résultats dans le simulateur de croissance Fagacée a été faite. 


\section{Relations entre morphologie, croissance, bois de réaction et contraintes de maturation. Apport de la technologie LiDAR terrestre pour répondre à des questions écologiques et sylvicoles}

par Mathieu DASSOT

Thèse soutenue publiquement le 15 janvier 2013 à Nancy

Directeur de thèse : Mme Meriem Fournier (AgroParisTech, LERFoB) ; co-directeur de thèse : M. Thiéry Constant (INRA, LERFoB)

Du point de vue écologique, les efforts de tension ou de compression développés par la maturation du bois au cours de la croissance permettent à l'arbre de maintenir sa posture verticale face à la gravité. Ces efforts, appelés autocontraintes de maturation, résultent de la mise en place d'un bois particulier, le bois de réaction. Sur le plan mécanique, c'est la dissymétrie des autocontraintes entre les deux faces du tronc de l'arbre qui joue un rôle moteur dans le redressement, qui s'exprime par une courbure. Ce travail vise à développer de nouveaux outils théoriques et métrologiques pour étudier le lien entre morphologie de l'arbre et autocontraintes dans un cadre biomécanique et écologique.

Une première approche vise à mettre en relation la dynamique de croissance et la compétition du peuplement avec la morphologie et la réaction de contrôle de la posture des arbres. Elle utilise des données issues d'un essai sylvicole de long terme (plantations de hêtres de différentes densités initiales laissées en croissance pendant 26 ans). L'analyse rétrospective de la production du bois de réaction sur des rondelles prélevées sur ces arbres a permis d'évaluer les différentes composantes du mouvement gravitropique au cours du temps. Une loi d'échelle, établie entre la vitesse de courbure et la circonférence des tiges, montre l'effet prépondérant du diamètre de la tige dans sa capacité de réaction, et l'absence d'effet additionnel de la compétition. En fin d'expérience, l'évaluation de la morphologie des arbres a permis de confirmer le lien entre l'inclinaison et l'élancement de la tige avec les indicateurs de contraintes de croissance.

D'un point de vue mécanique, la morphologie de l'arbre s'interprète par la forme de sa tige (inclinaison et courbures), et par la distribution spatiale de sa biomasse, qui peuvent fournir des variables biomécaniques candidates pour élaborer des modèles de stimulus-réponse. Ainsi, un important travail méthodologique couplant la technologie lidar terrestre à des techniques de modélisation géométrique a permis d'obtenir des maquettes 3D précises de la structure ligneuse d'arbres de différentes espèces. Les maquettes ont permis de modéliser la contrainte de flexion exercée par la biomasse aérienne des arbres en vue d'une mise en relation avec les indicateurs de contraintes de croissance mesurées sur leur grume. Les résultats montrent que la contrainte de flexion est une variable très prometteuse pour évaluer le degré de réaction des arbres. La méthodologie ouvre également des perspectives originales pour le suivi temporel de la morphologie de l'arbre en lien avec son interprétation biomécanique.

\section{Apport du multi-traçage isotopique $(26 \mathrm{Mg}, 44 \mathrm{Ca}$ et $2 \mathrm{H})$ à la connaissance des flux d'éléments minéraux dans les écosystèmes forestiers}

par Gregory van der HEIJDEN

Thèse soutenue publiquement le 4 février 2013 à Champenoux

Directeur de thèse : M. Étienne Dambrine (INRA-Université de Savoie, UMR CARRTEL) ; co-encadrant : M. Arnaud Legout (INRA, UR BEF)

De nombreux sols forestiers français sont acides et pauvres en éléments nutritifs et les forêts françaises font face aujourd'hui à des pressions extérieures croissantes. Ces nouvelles contraintes sont d'ordre nutritionnel, sylvicole ou climatique et risquent d'impacter la durabilité des écosys- 
tèmes forestiers à faible fertilité minérale. Il est donc très important de comprendre et quantifier les processus qui régissent les cycles des éléments nutritifs dans ces écosystèmes afin de mieux prédire leurs évolutions possibles face à de telles contraintes et aider les gestionnaires forestiers à prendre des décisions réfléchies pour assurer leur durabilité.

L'objectif principal de cette thèse était de comprendre le cycle du calcium et du magnésium dans un écosystème forestier productif bien que croissant sur un sol très faiblement pourvu en $\mathrm{Ca}$ et Mg. Pour cela, nous avons établi dans un peuplement de Hêtre âgé de 35 ans des bilans de flux entrées-sorties basés sur des mesures classiques de pools et flux entre 2003 et 2008. Puis une expérience de multitraçage isotopique a ensuite été conduite : les traceurs ont été apportés à la surface du sol en avril 2010 sous forme soluble et leur progression a été suivie dans l'écosystème jusqu'en mars 2012.

Les résultats ont mis en évidence le rôle primordial que joue la matière organique dans les cycles du Mg et du Ca, en leur conférant notamment un caractère très conservatif. Le $\mathrm{Mg}$ et le Ca apportés ont été captés dans la fine couche de litière au sol puis lentement libérés. Dans les horizons minéraux, la matière organique du sol, qui semble contribuer à la plus grande part de la capacité d'échange cationique du sol, a fortement contribué à ralentir la migration du $\mathrm{Mg}$ et du Ca à travers le sol. Par rapport au $\mathrm{Mg}$, le Ca apporté a été plus intensément retenu dans la litière, et moins intensément lixivié à travers le sol en raison de sa plus grande affinité pour la matière organique.

Aucune perte par drainage de Mg ou Ca n'a été mesurée. Les traceurs, rapidement absorbés par les racines fines, ont migré dans l'arbre à des vitesses très différentes : les feuilles étaient enrichies en $\mathrm{N}$ dès le mois suivant l'apport des traceurs, tandis que seuls de très faibles enrichissements en Mg ont été mesurés dans la canopée en 2012.

L'utilisation de traceurs isotopiques a permis de mettre en évidence la lenteur de la redistribution de $\mathrm{Mg}$ et encore plus de Ca dans l'écosystème, d'une part de la litière vers les sols et d'autre part des racines vers les canopées.

Enfin, la dilution isotopique mesurée dans les différents compartiments de l'écosystème à l'issue de deux années a permis de dresser des bilans et ainsi de valider les pertes de $\mathrm{Mg}$ calculées par l'approche classique de "bilan de fertilité minérale ». En revanche, les pertes de Ca calculées par cette même approche ont été mises en défaut, démontrant la contribution d'une source externe (absorption par les feuilles, altération) ou interne (mobilisation du stock des tissus ligneux), non prise en compte dans l'approche classique.

L'ensemble de ces travaux souligne l'intérêt de l'approche par traçage isotopique en complément des approches conventionnelles afin d'étudier la fertilité minérale des écosystèmes forestiers. 